\title{
Artgerecht? - Haltung von kleinen Heimtieren
}

\author{
Barbara M. Schneider
}

Kleine Heimtiere können für Kinder und Erwachsene eine Bereicherung darstellen. Doch worauf kommt es an, damit sich die Tiere wirklich wohlfühlen und nicht krank werden?

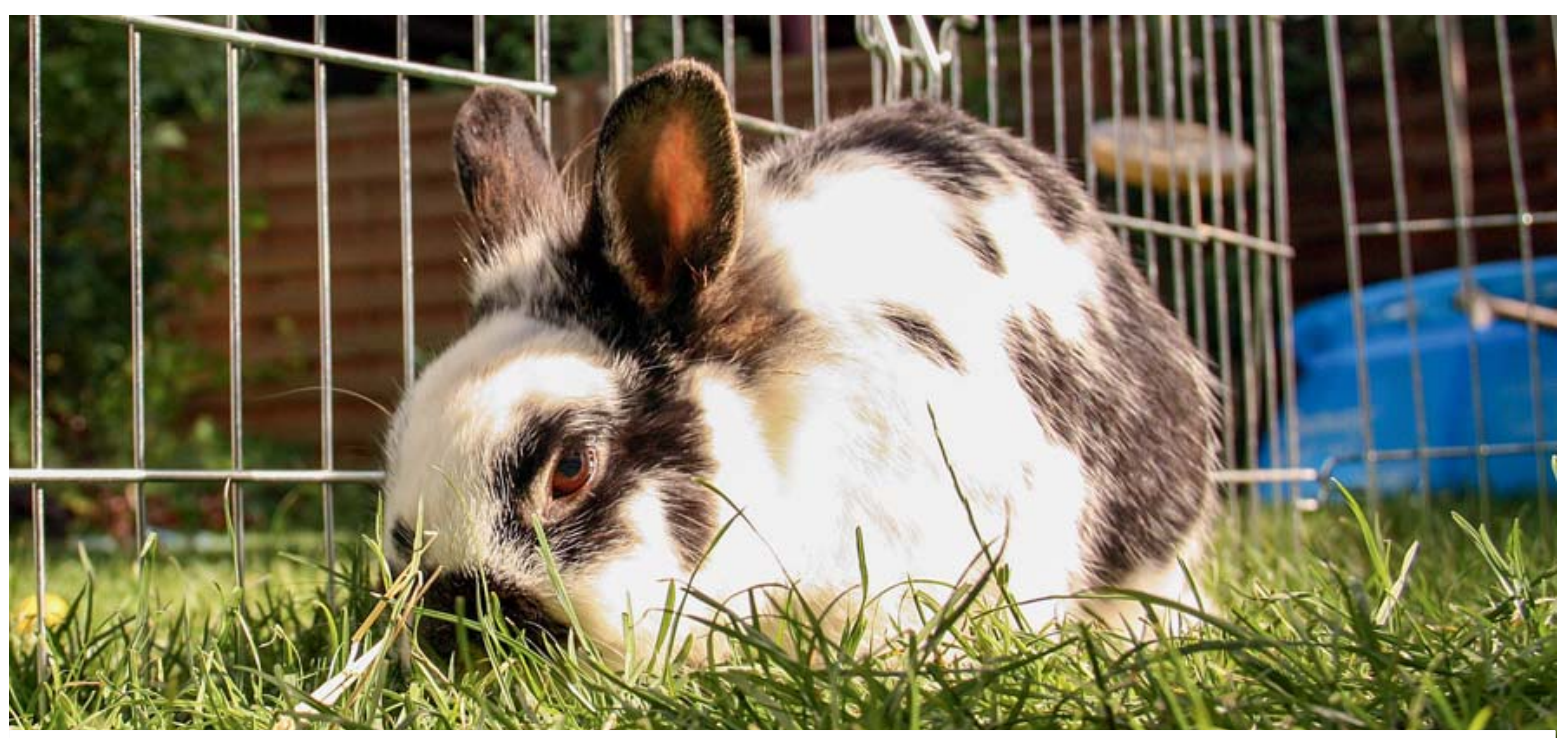

Quelle: Kirsten Oborny

\section{Die Bedürfnisse lassen sich aus dem Normalverhalten ableiten}

Um im Tierschutzgesetz festgelegten Forderungen nachkommen zu können, ist ein solides Wissen um das Normalverhalten der jeweils gehaltenen Spezies zwingend notwendig. Aus dem Normalverhalten lassen sich die Bedürfnisse einer Tierart für die unterschiedlichen Funktionskreise ablesen. Eine Haltung kann nur angemessen sein, wenn neben den Grundbedürfnissen wie Wasser und Futter auch die Bedürfnisse in Bezug auf Sozialkontakt, Rückzugsmöglichkeiten, Bewegung, Beschäftigung etc. befriedigt werden können. Werden diese auf Dauer nicht oder nur teilweise erfüllt, ist die Haltung als tierschutzwidrig anzusehen.

\section{Ernährung an das natürliche Nahrungsangebot anpassen}

Grundsätzlich muss das Nahrungsangebot im natürlichen Lebensraum der Tiere als Grundlage für die Erstellung einer artgerechten Ration verwendet werden. Wichtig ist beispielsweise, ob die Tiere insgesamt an karge Nahrung angepasst sind, eher Körner und Sämereien oder Gras verspeisen oder auch ab und zu tierisches Protein zu sich nehmen.

Wildkaninchen sind beispielsweise auf das Fressen von Gräsern spezialisiert ( $>$ Abb. 1). Sie nehmen Halme einzeln auf und zerkauen sie Schritt für Schritt mit bis zu 120 Kauschlägen pro Minute [2]. Da Gras eine nährstoffund kalorienarme Nahrungsquelle darstellt, sind wildlebende Kaninchen viele Stunden täglich mit der Nahrungsaufnahme beschäftigt. Das gesamte Verdauungssystem der Kaninchen ist an diese Art der Nahrung angepasst. Durch das Kauen beim Verzehr einzelner Gras- oder Heu- 
halme werden aber nicht nur die beständig nachwachsenden Zähne am besten abgerieben. Das Kauen hat zudem einen beruhigenden Effekt auf die Tiere. Es gibt Studien, die deutlich zeigen, dass eine Heufütterung Stereotypien wie Gitternagen, exzessive Fellpflege und auch Fellfressen signifikant reduziert [3-5].

Nicht nur das Futter an sich, sondern auch die Art der Futteraufnahme ist entscheidend, wenn alle damit verbundenen Bedürfnisse befriedigt werden sollen. Bei Tierarten, die sich ihr Futter mühsam zusammensuchen müssen (z. B. Goldhamster, Wüstenrennmäuse), ist es beispielsweise wichtig, dass auch in der Heimtierhaltung das Futter gesucht und erarbeitet werden muss und nicht einfach in einem Napf vorgelegt wird. Chinchillas nehmen beispielsweise ihr Futter in die Vorderpfoten, untersuchen es und machen einen Probebiss. Angebotene Futterbestandteile müssen über eine entsprechende Größe verfügen, um dies zu ermöglichen.

\section{Was ist bei der Gruppenhaltung zu beachten?}

Das Sozialverhalten bildet eine weitere wichtige Grundlage für eine tiergerechte Haltung. Werden hier Fehler gemacht, kann dies schnell zu chronischem Stress und damit verbundenen Problemen führen. Nicht selten hat dies auch einen negativen Einfluss auf die Lebenserwartung.

Einige Tierarten wie Meerschweinchen, Ratten oder Kaninchen sind obligat sozial. Das bedeutet, dass sie zwingend mit Artgenossen zusammenleben müssen, um sich wohlzufühlen. Allein gehaltene Tiere dieser Arten leiden. Andere Spezies können den arteigenen Sozialpartner ebenfalls nicht ersetzen, da sie auf eine andere Art kommunizieren und andere Bedürfnisse haben. Die bedauerlicherweise immer noch häufig praktizierte gemeinsame Haltung eines einzelnen Meerschweinchens und eines einzelnen Kaninchens ist somit nicht tiergerecht und kompromisslos abzulehnen.

Eine gemeinsame Haltung unterschiedlicher Tierarten ( $\triangleright$ Abb. 2) ist nur dann akzeptabel, wenn ...

- die jeweiligen Tierarten in Gruppen gehalten werden,

- der Platz ausreicht, sodass sie sich aus dem Weg gehen können,

- die Bedürfnisse in Bezug auf Nahrungsaufnahmeverhalten und

- die Rückzugsmöglichkeiten jederzeit gewährleistet werden können.

Doch auch bei der Gruppenhaltung sozialer Tierarten gilt es Einiges zu beachten, damit das Zusammenleben harmonisch verläuft. Wie eine Gruppe zusammengestellt sein muss, damit es möglichst wenig Probleme gibt, ist

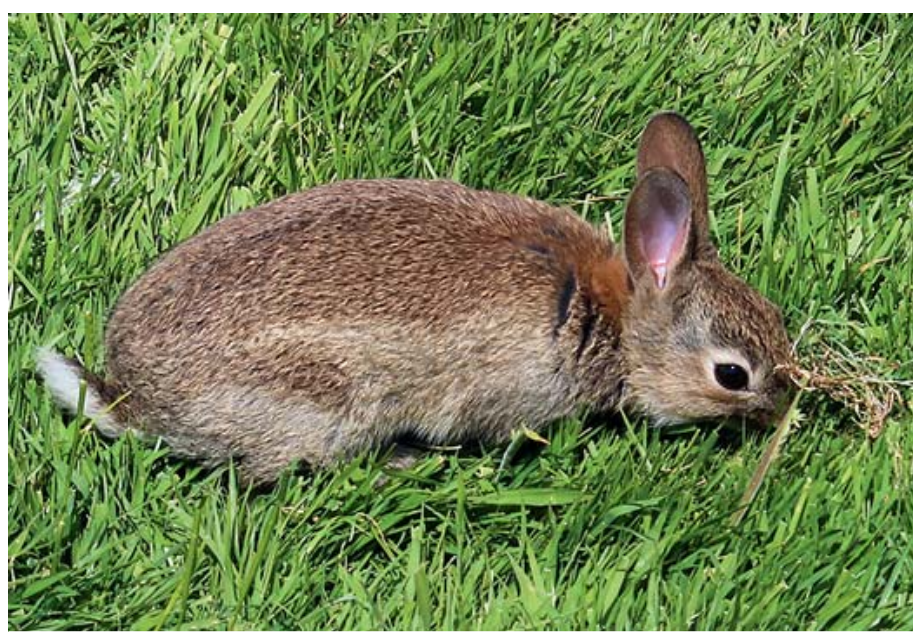

- Abb. 1 Wildkaninchen beim Fressen von Gras auf der Wiese. Quelle: Barbara Schneider

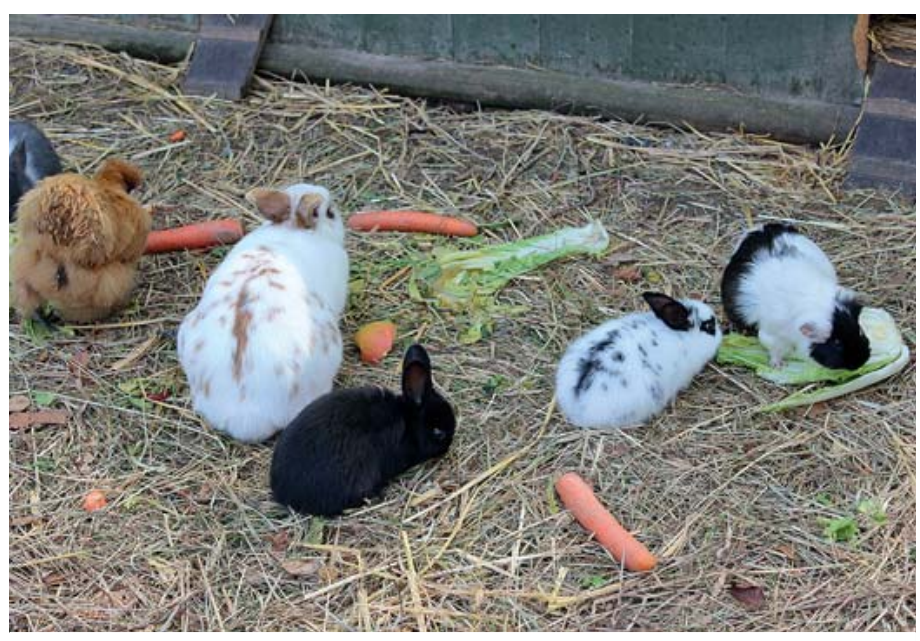

- Abb. 2 Eine gemeinsame Haltung unterschiedlicher Tierarten ist nur möglich, wenn u. a. diese in Gruppen gehalten werden, der Platz ausreicht und die Bedürfnisse in Bezug auf Nahrungsaufnahmeverhalten und Rückzugsmöglichkeiten erfüllt werden. Quelle: Barbara Schneider

WER EIN TIER HÄLT, BETREUT ODER ZU BETREUEN HAT ... Im Tierschutzgesetz [1] ist unter $\S 2$ festgelegt, dass jede Person, die ein Tier hält, betreut oder zu betreuen hat, dieses seiner Art und seinen Bedürfnissen entsprechend angemessen ernähren, pflegen und verhaltensgerecht unterbringen muss. Sie darf zudem die Möglichkeit des Tieres zu artgemäßer Bewegung nicht so einschränken, dass ihm Schmerzen, vermeidbare Leiden oder Schäden zugefügt werden. Darüber hinaus muss sie über die für eine angemessene Ernährung, Pflege und verhaltensgerechte Unterbringung des Tieres erforderlichen Kenntnisse und Fähigkeiten verfügen. 


\section{GEWÖHNUNGSTRAINING}

Das Gewöhnungstraining wird bei allen kleinen Heimtierarten ähnlich durchgeführt. Grundsätzlich sollten alle beteiligten Tiere immer entspannt bleiben, Auseinandersetzungen zwischen ihnen müssen vermieden werden. Die beiden Tiere, die aneinander gewöhnt werden sollen, müssen jeweils in getrennten, tiergerechten Haltungseinheiten mit ausreichend Rückzugsmöglichkeiten gehalten werden.

\section{Schritt 1: Geruchsaustausch}

Die Haltungseinheiten werden anfangs so nahe nebeneinander gestellt, dass die Tiere gerade nicht aufeinander reagieren und ruhig bleiben. Einmal bis mehrmals täglich muss eine Handvoll benutzte Einstreu zwischen den Käfigen ausgetauscht werden. Dies fördert den Geruchsaustausch und die Bildung eines Gruppengeruchs.

\section{Schritt 2: Annäherung}

Die Haltungseinrichtungen werden einander nach und nach angenähert. Auch hier ist wiederum darauf zu achten, dass die Tiere nicht aufeinander reagieren und keine Stresszeichen zeigen. Falls dies der Fall ist, müssen die Gehege wieder weiter getrennt und die Annäherung sollte beim nächsten Versuch langsamer durchgeführt werden.

Zudem sollten immer besonders begehrte Leckerchen in Anwesenheit des anderen Tieres verfüttert werden, um eine Gegenkonditionierung zu erreichen.

\section{Schritt 3: Erster Direktkontakt}

Wenn beide Haltungseinheiten über mehrere Tage direkt nebeneinander stehen können, ohne dass die Tiere aufeinander reagieren, kann das erste Zusammenführen erfolgen. Dies muss immer auf sogenanntem neutralen Terrain geschehen, also in einem Areal, das keines der beiden Tiere kennt. Hier sollte es viele Rückzugs- und Versteckmöglichkeiten (immer mit 2. Ausgang) geben, sodass sich die Tiere aus dem Weg gehen können. Sackgassen müssen vermieden werden. Alle ersten Begegnungen der Tiere müssen unter aktiver Überwachung stattfinden, damit ein rechtzeitiges Eingreifen und Abbrechen möglich ist. Wenn beide Tiere gut miteinander auskommen, wird schrittweise die Zeit verlängert, in der sie gemeinsam auf neutralem Boden Auslauf haben.

\section{Schritt 4 Umzug}

Erst wenn mehrere Tage hintereinander ein gemeinsamer Auslauf für längere Zeit problemlos möglich ist, können die Tiere in eine gemeinsame Haltungseinheit gegeben werden, die frisch gesäubert sein muss und nicht nach nur einem der beiden Tiere riechen darf.

dabei von Tierart zu Tierart unterschiedlich. Die beste Haltungsform für Kaninchen ist beispielsweise eine gemischte Gruppe mit einem kastrierten Männchen und 2 Weibchen oder 2 kastrierten Männchen mit 2-4 Weibchen [6]. Eine Vergesellschaftung erfolgt bei allen Tierarten idealerweise bereits im Jungtieralter. Bei älteren
Tieren sollte sie immer nur nach einem Gewöhnungstraining stattfinden, bei dem die Tiere sich langsam an Geruch, Anblick und Geräusche des neuen Partners gewöhnen können.

Nur wenige der häufig als Haustier gehaltenen Heimtiere sind Einzelgänger. Als bekanntestes Beispiel ist hier der Goldhamster zu nennen. Werden solche Tierarten in Gruppen gehalten, sind schwere Aggressionen und Verletzungen vorprogrammiert.

\section{Rückzugsmöglichkeiten helfen, unnötigen Stress zu vermindern}

Auch in Bezug auf die notwendigen Rückzugs- und Versteckmöglichkeiten ist wieder das Normalverhalten der wildlebenden Verwandten zugrunde zu legen ( $\bullet$ Abb. $\mathbf{3}$ ). Da es sich bei den kleinen Heimtieren um Beutetiere handelt, die mitunter nicht sehr wehrhaft sind, sind tiergerechte Rückzugsmöglichkeiten essenziell für ihr Wohlbefinden. Wenn sie sich bei (vermeintlicher) Gefahr nicht verstecken können, sind sie unnötigem Stress ausgesetzt ( Abb. 4a).

Grundsätzlich müssen in einem Gehege ausreichend Unterschlupfmöglichkeiten zur Verfügung stehen, damit alle Tiere gleichzeitig Zuflucht finden können ( Abb. 4b). Als Faustregel muss für jedes Individuum ein Häuschen zum Rückzug zur Verfügung stehen. Nur dann können sich die Tiere alle in Sicherheit begeben und dennoch aus dem Weg gehen, wenn nötig. Zudem sollte es in den Häuschen immer einen 2. Ausgang geben, damit es darin nicht zur Bildung einer Sackgasse kommt und Auseinandersetzungen besser vermieden werden können.

Auch bei der Gestaltung von Auslauf- und Freilaufmöglichkeiten muss dem Bedürfnis nach einer angemessenen Rückzugsmöglichkeit Rechnung getragen werden. Sonst kann es dazu kommen, dass der angebotene Platz nicht genutzt wird, da er den Tieren nicht sicher erscheint. Nicht selten wird eine mangelnde Nutzung eines schlecht strukturierten Auslaufs von Besitzern dahingehend interpretiert, dass die Tiere diesen grundsätzlich nicht benötigen.

\section{Gehegegröße an Bewegungsbedürfnisse anpassen}

Kleine Heimtiere haben trotz ihrer geringen Größe in der Regel ein ausgeprägtes Bewegungsbedürfnis. Dies liegt unter anderem daran, dass sie in der Wildnis oft einen Teil des Tages mit der Suche nach Futter beschäftigt sind und dabei unter Umständen weite Strecken zurücklegen müssen. Ist das Gehege nicht ausreichend groß, und sind kei- 


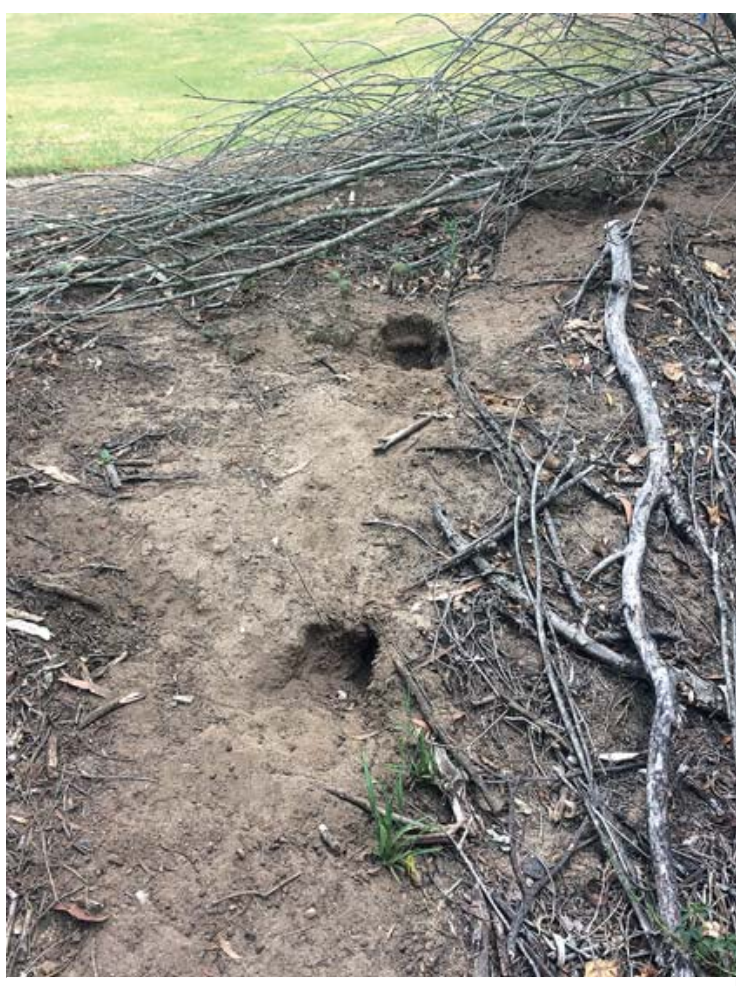

- Abb. 3 Selbstgegrabener Bau von Wildkaninchen: Bei Gefahr ziehen sich die Tiere in diese dunklen, sicheren Unterschlüpfe zurück. Quelle: Barbara Schneider

\section{LAUFRAD}

Anforderungen an ein tiergerechtes Laufrad:

- ohne Farbüberzug

- achsseitig geschlossen

- keine Querstreben

- geräuschfrei

- Durchmesser so groß, dass das Tier ohne Lordose laufen kann

ne weiteren Bewegungsanreize wie beispielsweise Strukturen, Laufräder oder Laufteller gegeben, kann es zu Problemen kommen.

Typische Probleme sind (nach Rothfritz [7]):

- Adipositas

- stereotypes Verhalten

- Knochengewebshypoplasien

- Wirbelsäulenverkrümmungen

Die überwiegende Zahl der kommerziell erhältlichen Käfige ist zu klein, um eine tiergerechte Haltung zu gewährleisten. So benötigen beispielsweise 2-3 Kaninchen eine Mindestfläche von $6 \mathrm{~m}^{2}$ und jedes weitere Tier zusätzliche $2 \mathrm{~m}^{2}$ [8]. Selbst gebaute Gehege oder großzügige Außengehege sind in der Regel am tiergerechtesten $(\triangleright$ Abb. 5).
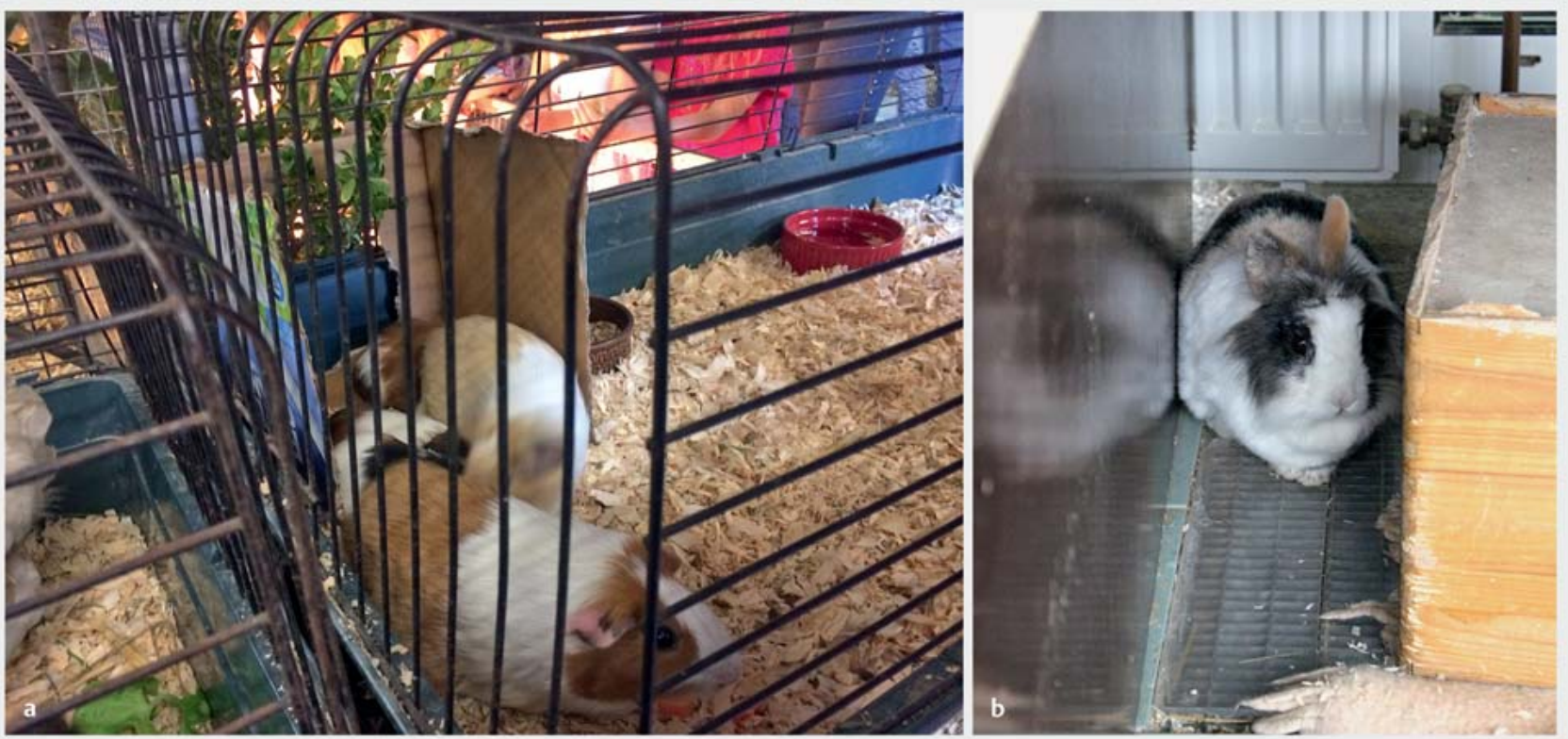

- Abb. 4 a Die sichtbar gestressten Meerschweinchen drängen sich um den provisorischen Unterschlupf, der ihnen in diesem Käfig die einzige Sicherheit bietet. b Versteckversuch eines Kaninchens neben dem Unterschlupf bei Überbelegung des Käfigs. Quelle: Barbara Schneider 


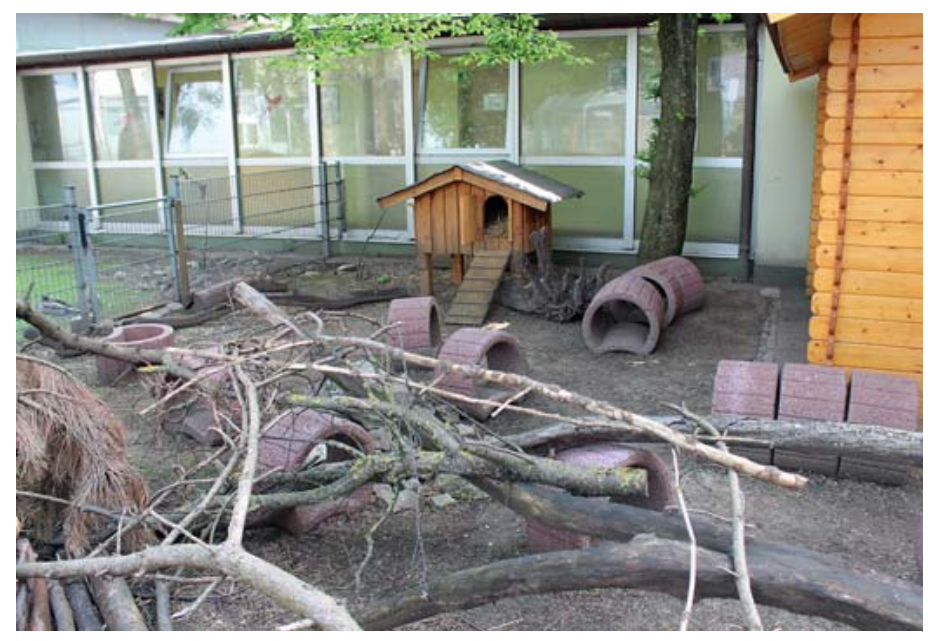

- Abb. 5 Einer Gruppe von 2 Kaninchen sollte mindestens ein $6 \mathrm{~m}^{2}$ großes, strukturiertes Gehege zur Verfügung stehen, wie in diesem Außengehege eines Tierheims gegeben. Quelle: Barbara Schneider

\section{Individuelle Bedürfnisse bei der Gehegegestaltung beachten}

Doch nicht nur die Größe des Auslaufs ist entscheidend, auch die Strukturierung und Gestaltung der Haltungseinheit ist wichtig. So wird, wie bereits erwähnt, die zur Verfügung stehende Fläche unter Umstünden nur dann genutzt, wenn ausreichend Versteckmöglichkeiten darin verteilt sind, sodass sich die Tiere sicher fühlen. Viele der gängigen kleinen Heimtiere zeigen nämlich eine ausgeprägte Thigmotaxis. Dies bedeutet, dass sie sich vor allem in direkter Nähe von Strukturen wie Häuschen, Wänden oder anderen Gegenständen aufhalten und offene Flächen gemieden werden. Zudem ist eine Haltungseinheit interessanter und regt zu mehr Bewegung an, wenn sie nicht von einem Ort aus vollständig überblickt werden kann.

Aber nicht nur Laufen, Springen oder Klettern sind zum Bewegungsverhalten zu zählen. Viele der häufig gehaltenen kleinen Heimtiere verfügen zusätzlich über ein ausgeprägtes Grabebedürfnis. Bei Hamstern, Gerbils etc. muss daher auch ein tief eingestreuter Bereich zur Verfügung stehen, in dem die Tiere diesem Bedürfnis nachkommen und sich eigene Bauten graben können. Doch auch Degus, Ratten, Mäuse und Kaninchen graben gerne und sollten zumindest eine Buddelkiste zur Verfügung gestellt bekommen. Außengehege müssen entsprechend in der Tiefe gegen ein Graben nach draußen gesichert sein.

\section{Tiergerechte Beschäftigung zur Stressreduktion}

Eine Buddelkiste befriedigt nicht nur das Grabebedürfnis der kleinen Heimtiere, sondern auch das Bedürfnis nach Abwechslung. Diese wird am besten durch eine Anreicherung der Haltungsumwelt (Enrichment) mit verschiedenen Reizen und Strukturen erzielt. Dieses dient nicht nur der artgemäßen Beschäftigung der Tiere, sondern erfüllt zudem weitere Zwecke. Werden verschiedene Reizqualitäten (z. B. weich - hart, warm - kühl) angeboten, haben die Tiere die Möglichkeit, zwischen diesen zu wählen. Auf diese Weise behalten sie eine gewisse Kontrolle über ihre Haltungsumwelt, was wiederum hilft, Stress zu reduzieren. Ist eine Haltung entsprechend strukturiert, so erlaubt das auch, die Verhaltensweisen unterschiedlicher Funktionskreise an unterschiedlichen Orten auszuführen, also beispielsweise den Ausscheidungs- vom Ruheort zu trennen. Dieses Bedürfnis nach unterschiedlichen Funktionsbereichen macht man sich u. a. beim Toilettentraining der Kaninchen zunutze.

\section{TOILETTENTRAINING FÜR KANINCHEN}

Beim Toilettentraining beginnt man in einem kleinen Areal und stellt Toiletten an Orten auf, an denen das Kaninchen ohnehin bevorzugt ausscheidet. Normalerweise ist das u. a. unter der Heuraufe. Es empfiehlt sich, etwas Kot und Urin in die Toilette zu legen, sodass diese auch als solche besser erkannt wird. Wenn die Ausscheidung zuverlässig in den Toiletten klappt, kann man Schritt für Schritt größere Areale zugänglich machen. Es sollten immer viele Toiletten aufgestellt sein, damit die Kaninchen nicht zu weit dorthin laufen müssen. An allen bevorzugten Ausscheidungsorten sollte eine aufgestellt werden. Selbstverständlich sollten Ausscheidungen außerhalb der Toilettenschalen niemals bestraft, sondern jede Benutzung der Toiletten gelobt werden.

Es gilt:

- in einem kleinen Areal anzufangen

- mehrere Toiletten aufzustellen

- die Toilette in die Ausscheidungsecke des Käfigs zu stellen

- den Kot in die Toilette zu legen

- den Urin mit Papier aufzunehmen und in die Toilette zu legen

- langsam mehr Raum zugänglich zu machen

- Toiletten an bevorzugte Orte zu stellen

- Tiere zu loben

Auch der Freilauf außerhalb des Geheges kann Enrichment darstellen. Bei diesen Gelegenheiten können die Tiere neues Terrain und neue Strukturen erkunden. Gerade für die besonders neugierigen Tierarten wie Ratten, 
Mäuse, aber auch Kaninchen ist dies eine willkommene Abwechslung.

Für Kaninchen sind die wichtigsten Enrichment-Elemente

( $\triangleright$ Abb. 5):

- Unterschlüpfe

- erhöhte Ebenen als Aussichtspunkte

- rohfaserreiches Futter

- Nagemöglichkeiten

\section{Umgang mit kleinen Heimtieren}

Im Zuge einer tiergerechten Haltung ist auch auf einen tiergerechten Umgang zu achten. Grundvoraussetzung dafür ist unter anderem, dass die Tiere langsam und mit viel positiver Bestärkung (Leckerchen) an die Anwesenheit des Menschen und das notwendige Handling gewöhnt werden. Besonders bei scheuen Tieren muss hier sehr langsam vorgegangen werden. Anfangs wird eventuell nur die ruhige Anwesenheit eines Menschen im selben Raum toleriert. Wenn das Tier entsprechend ruhig bleibt, kann Schritt für Schritt eine weitere Annäherung erfolgen. Hier wird wieder - ähnlich wie beim Gewöhnungstraining - im Tempo des jeweiligen Tieres vorangeschritten.

Aber auch bei Tieren, die das Handling gewöhnt sind, muss immer die tierschonendste Methode zum Fangen und Hochheben verwendet werden. Viele kleine Heimtiere laufen beispielsweise relativ problemlos in höhlenartige Strukturen (Hände, Becher, Röhren), mit deren Hilfe sie im Anschluss hochgehoben werden können. Grundsätzlich muss jegliches Handling vermieden werden, das mit Schmerzen für die Tiere verbunden ist oder ihnen Unwohlsein bereitet. Das bedeutet, dass Tiere nicht nur an einzelnen Körperteilen wie beispielsweise Ohren oder Schwanz hochgehoben werden dürfen. Es muss immer der gesamte Körper beim Hochheben gestützt werden. Besonders bei Tieren ab der Größe einer Ratte sind dazu in der Regel 2 Hände vonnöten. Auf die korrekte Anleitung und Unterstützung von Kindern im Hinblick auf das Handling muss besonderen Wert gelegt werden ( A Abb. 6).

Speziesspezifische Besonderheiten müssen ebenfalls beachtet werden. So sollte beispielsweise das Handling eines Hamsters nach Möglichkeit tagsüber vermieden werden oder ein Meerschweinchen nicht gestreichelt werden. Da Meerschweinchen dies nicht mögen, sind sie beispielsweise nur für solche Kinder geeignet, die gerne beobachten. Auch die häufig anzutreffende Methode, dass Meerschweinchen nachts in ein anderes Zimmer verbracht werden, ist aus Sicht eines Meerschweinchens nach Möglichkeit zu vermeiden, da diese Tiere nicht gut auf Veränderungen reagieren.

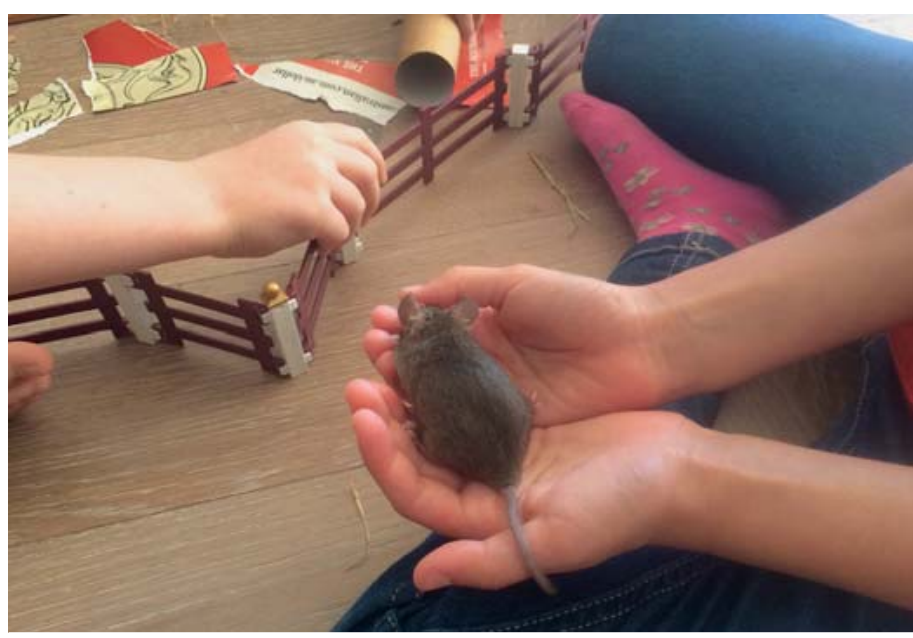

- Abb. 6 Kinder müssen im Umgang mit kleinen Heimtieren angeleitet werden. Quelle: Barbara Schneider

\section{Sollten weitere Besonderheiten beachtet werden?}

Bei der Haltung von kleinen Heimtieren sind weitere Besonderheiten zu bedenken. Das Hörvermögen ist beispielsweise bei den meisten als Haustiere gehaltenen Arten deutlich unterschiedlich zu dem des Menschen. Auch die Lärmempfindlichkeit ist im Allgemeinen höher. Auf eine möglichst ruhige Umgebung ist daher zu achten.

Da die Heimtiere in der Regel Beutetiere sind, reagieren sie in bestimmten Situationen besonders schreckhaft. Plötzliche Bewegungen von oben lösen besonders häufig Stress und/oder Flucht- oder Erstarr-Reaktionen aus. Sie sind daher zu vermeiden.

\section{TAKE HOME}

Folgende Punkte sind in Hinblick auf eine tiergerechte Haltung besonders zu beachten:

- artgerechte Nahrung

- Berücksichtigung speziesspezifischen Sozialverhaltens

- Rückzugsmöglichkeiten für alle Tiere, über den gesamten Lebensraum verteilt

- große, gut strukturierte Gehege

- Enrichment

- tiergerechtes Handling

\section{Fazit}

Kleine Heimtiere sind beliebte Haustiere. Dennoch ist ihre Haltung zum Teil recht anspruchsvoll und erfordert ein solides Wissen um das Normalverhalten und die Be- 
dürfnisse der jeweiligen Tierart. Werden diese Bedürfnisse jedoch befriedigt, können die Tiere hervorragende Begleiter sein.

\section{Korrespondenzadresse}

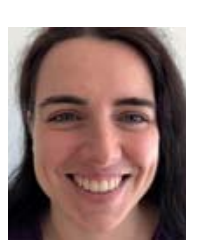

Dr. Barbara M. Schneider

Fachtierärztin für Verhaltenskunde

Graf-Moy-Str. 2

85465 Freising

b.schneider@verhalten-schneider.de

www.verhalten-schneider.de

\section{Literatur}

[1] Tierschutzgesetz. Im Internet: https://www.gesetze-iminternet.de/tierschg/TierSchG.pdf; Stand: Januar 2019

[2] Bradley Bays T. Rabbit behavior. In: Bays T, Lightfoot T, Mayer J, eds. Exotic Pet Behavior: Birds, Reptiles, and Small Mammals. St. Louis: Saunders Elsevier; 2006

[3] Berthelsen H, Hansen LT. The effect of hay on the behavior of caged rabbits (Oryctolagus cuniculus). Anim Welfare 1999; 8: 149-157
[4] Lidfors L. Behavioural effects of environmental enrichment for individually caged rabbits. Appl Anim Behav Sci 1997; 52: 157-169

[5] Mulder A, Nieuwenkamp AE, van der Palen JGP et al. Supplementary hay reduces fur chewing in rabbits. Tijdschrift Diergeneeskd 1992; 117: 655-658

[6] Morgenegg R. Artgerechte Haltung - ein Grundrecht auch für (Zwerg-)Kaninchen. 3. Aufl. Bozen: tbv Tierbüerchverlag; 2000

[7] Rothfritz P, Loeffler K, Drescher B. Einfluss unterschiedlicher Haltungsverfahren und Bewegungsmöglichkeiten auf die Spongiosastruktur der Rippen sowie Brust- und Lendenwirbel von Versuchs- und Fleischkaninchen. Tierärztl Umschau 1992; 47: 758-768

[8] Döring D. Kaninchen. In: Schneider B, Döring D, Hrsg. Verhaltensberatung bei kleinen Heimtieren - Haltung, Normalverhalten und Behandlung von Verhaltensproblemen. Stuttgart: Schattauer; 2016

Bibliografie

DOI https://doi.org/10.1055/a-1091-0269

Kleintier konkret 2020; 23: 18-24

(c) Georg Thieme Verlag KG Stuttgart · New York ISSN 1434-9132 